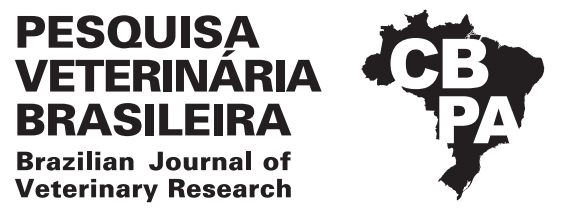

Pesq. Vet. Bras. 38(10):2006-2011, outubro 2018 DOI: $10.1590 / 1678-5150-P V B-5413$

Artigo Original

ISSN 0100-736X (Print)

ISSN 1678-5150 (Online)

\title{
Avaliação morfofuncional do corpo lúteo para diagnóstico precoce de gestação 20 dias após IATF em vacas mestiças leiteiras ${ }^{1}$
}

\author{
Mariana A.A. Silva ${ }^{2 *}$, Alexandra S. Rodrigues ${ }^{2}$, Tiago O. Brandão², \\ Luiz Di Paolo Maggitti Junior², Bia S.S. Carôso², Marcos Chalhoub ${ }^{2}$, \\ Rodrigo F. Bittencourt ${ }^{2}$ e Antonio L. Ribeiro Filho ${ }^{2}$
}

\begin{abstract}
Silva M.A.A., Rodrigues A.S., Brandão T.O., Maggitti Junior L.P., Carôso B.S.S., Chalhoub M., Bittencourt R.F. \& Ribeiro Filho A.L. 2018. [Morphological evaluation of corpus luteum for the diagnosis of 20-day pregnancy after IATF in crossbred cows.] Avaliação morfofuncional do corpo lúteo para diagnóstico precoce de gestação 20 dias após IATF em vacas mestiças leiteiras. Pesquisa Veterinária Brasileira 38(10):2006-2011. Departamento de Anatomia, Patologia e Clínicas Veterinárias, Universidade Federal da Bahia, Rua Ademar de Barros 500, Ondina, Salvador, BA 40170-110, Brazil. E-mail: marianadeandradesilva@gmail.com

The super early gestation detection is of great relevance considering the increase of the herds' productivity, in an attempt to reduce the time of service of these animals. Due to this relevance, this study aimed to use Doppler ultrasonography as a tool for gestation prediction, evaluating the luteal body (CL) irrigation twenty days after FTAI. 73 crossbred cows were evaluated 20 days after FTAI through trans rectal US. In order to obtain the diameter of the CL (DCL) and its area (ACL), B mode was used. The Color Doppler was applied to measure the vascularization area (CLV) and its percentage in the CL (VCL \%). The images were classified, based on the number of colored pixels and their distribution in CL, in two groups: negative predictive diagnosis of gestation (NPD) and positive predictive value (PPV). On the same day, LC functionality was determined based on $\mathrm{P} 4$ plasma concentration. The definitive gestation diagnosis was confirmed through US 35 days after the FTAIs. Student's T test (with P<0.05) was used to evaluate the variables. Pregnant group presented higher averages $(\mathrm{P}<0.05)$ for all variables compared to the Non-Pregnant Group. The predictive diagnosis showed 83.33\% of specificity, $100 \%$ of sensitivity and $91.79 \%$ of accuracy. The conclusion is that the US Doppler at 20 days post FTAI is a reliable method for early gestation diagnosis.
\end{abstract}

INDEX TERMS: Corpus luteum, 20-day pregnancy, diagnosis, IATF, cows, doppler, progesterone, vascularization, pregnancy prediction, morphology.

RESUMO.- A detecção superprecoce de gestação é de grande relevância quando se trata do aumento da produtividade dos rebanhos, na tentativa de redução do tempo de serviço desses animais. Por tal relevância, objetivou-se utilizar a ultrassonografia (US) Doppler como ferramenta para predição de gestação, avaliando a irrigação do corpo lúteo (CL), vinte dias após IATF. Setenta e três vacas mestiças foram avaliadas 20 dias após IATF por US transretal. Para obtenção do diâmetro do CL (DCL) e sua área (ACL) utilizou-se o modo

\footnotetext{
${ }^{1}$ Recebido em 27 de abril de 2017.

Aceito para publicação em 21 de dezembro de 2017.

2 Departamento de Anatomia, Patologia e Clínicas Veterinárias, Universidade Federal da Bahia (UFBA), Av. Ademar de Barros 500, Ondina, Salvador, BA 40170-110, Brasil. *Autor para correspondência: marianadeandradesilva@gmail.com
}

B, na mensuração da área de vascularização (VCL) e seu percentual no CL (VCL\%) empregou-se o Doppler Colorido. As imagens foram classificadas com base na quantidade de pixels coloridos e sua distribuição no CL, em dois grupos: diagnóstico preditivo negativo de gestação (DPN) e preditivo positivo (DPP). No mesmo dia, a funcionalidade do CL foi determinada pela concentração plasmática de $\mathrm{P} 4.0$ diagnóstico de gestação definitivo foi realizado por US 35 dias após as IATFs. Utilizou-se o teste t de Student (com $P<0,05)$ na avaliação das variáveis. Grupo gestantes apresentou médias superiores $(\mathrm{P}<0,05)$ para todas as variáveis ao Grupo Não Gestantes. O diagnóstico preditivo demonstrou 83,33\% de especificidade, sensibilidade de $100 \%$ e acurácia de $91,79 \%$. Conclui-se que a US doppler do CL aos 20 dias pós IATF é um método confiável no diagnóstico precoce de gestação. 
TERMOS DE INDEXAÇ̃̃O: Corpo lúteo, diagnóstico precoce, gestação 20 dias, IATF, vacas leiteiras, doppler, progesterona, vascularização, predição de gestação, morfologia.

\section{INTRODUÇÃO}

O corpo lúteo (CL) é uma glândula transitória, responsável pela produção de progesterona e inicia seu desenvolvimento no ovário logo após ovulação (Davis et al. 2003), é caracterizada como a estrutura que apresenta um dos maiores índices de vascularização por unidade de tecido no corpo (Acosta et al. 2002). Segundo Herzog et al. (2010), após ovulação, o tamanho do CL aumenta concomitantemente com a produção de progesterona, sendo correlacionado positivamente. Depois do desenvolvimento, o CL chega a uma fase estática de crescimento, no entanto, nesse período a concentração plasmática de progesterona continua aumentando assim como o fluxo sanguíneo para o CL (Bollwein et al. 2012). Um aumento dos índices de vascularização no CLé encontrado em fêmeas gestantes, sendo favorável à manutenção de altas concentrações de progesterona, já vacas não gestantes podem esboçar uma diminuição deste parâmetro após a ovulação (Herzog et al. 2011).

Wathes et al. (2003) descreveram em seu estudo que as vacas inseminadas após protocolo de Inseminação Artificial em Tempo Fixo (IATF), nas quais a gestação não se estabelece têm concentrações plasmáticas de progesterona mais baixas em comparação àquelas onde gestação é levada à termo. Nesse sentido, Mann \& Lamming (2001) evidenciaram que um atraso de um dia no aumento da progesterona pós-ovulação resultou em embriões que não atingiram a fase de alongamento e consequentemente falharam na liberação de interferon-tau no período do reconhecimento materno da gestação.

A ultrassonografia modo Doppler colorido pode possuir diversas vantagens aplicáveis na rotina reprodutiva, entre elas a detecção da não gestação precoce e a seleção de fêmeas para programas de sincronização e ressincronização do estro e da ovulação (Pinaffi et al. 2015). Essa ferramenta mostra a vascularização em um tecido, podendo ser utilizada com a finalidade de determinar o status fisiológico das estruturas ovarianas e uterinas (Herzog \& Bollwein 2007).

Neste contexto, variações nos níveis de irrigação do CL podem ser observadas entre gestantes e não gestantes a partir do $15^{\circ}$ dia após inseminação artificial (IA), onde a diminuição da irrigação poderia ser um indicativo de luteólise em animais não gestantes. No entanto, efeitos individuais não garantem uma elevada confiabilidade do diagnóstico de gestação realizado neste momento (Herzog et al. 2011), já que existe possibilidade de perda embrionária precoce ou formação de CL persistente (Siqueira et al. 2013).

Ginther et al. (2007) verificaram um aumento na fluxometria para o CL nos momentos próximos a luteólise o que poderia ser diagnosticado como falso positivo. Contudo para Siqueira et al. (2013) após 20 dias da IA, onde já existe um processo de luteólise mais evidente devido ao início de um novo ciclo estral, é possível observar maiores disparidades entre o fluxo sanguíneo de vacas gestantes e não gestantes, sendo este 0 momento mais adequado para realizar o diagnóstico precoce com a utilização da ferramenta Doppler. Após esse período, uma ovulação proveniente do próximo ciclo resultaria na formação de um corpo hemorrágico (Forde et al. 2011), o que tornaria a avaliação menos confiável.
Tendo em vista a demanda de mercado pela diminuição de intervalo entre partos visando maior produtividade, objetivou-se utilizar ultrassonografia Doppler como ferramenta para predição precoce da gestação, avaliando a irrigação do CL, 20 dias após IATF, assim como comparar a funcionalidade do CL entre vacas gestantes e não gestantes.

\section{MATERIAL E MÉTODOS}

O projeto do experimento foi submetido a avaliação da Comissão de Ética no Uso de Animais, da Universidade Federal da Bahia, sendo aprovado sob protocolo de número 52/2015.

Local e animais. 0 experimento foi realizado no município de Entre Rios, região Nordeste da Bahia, com latitude 11ำ56’31" sul, longitude $38^{\circ} 05^{\prime} 04^{\prime \prime}$ oeste, estando a uma altitude de 162 metros. A região apresenta pluviosidade média anual de $1.251 \mathrm{~mm}$, clima quente e semiúmido. A propriedade adotava um sistema extensivo de criação de animais em pastagem de Brachiaria decumbens, suplementação mineral e água ad libitum.

Foram utilizadas 73 fêmeas mestiças Bostaurustaurusx Bostaurusindicus, pertencentes a categoria multípara, com escore de condição corporal (ECC) $\geq 3$, utilizando-se escala de 1 a 5 (Houghton et al. 1990).

Previamente ao início da sincronização, os animais foram submetidos a um exame clínico, ginecológico e ultrassonografia transretal, utilizando-se um transdutor linear com frequência de 6MHz (Mindray Z5, Shenzhen, China), sendo consideradas aptas a participarem do experimento, fêmeas que apresentaram no momento da avaliação ausência de anormalidades no trato reprodutivo.

Todos os animais usados no experimento foram vacinados contra doenças da esfera reprodutiva e a propriedade obedecia ao calendário oficial estadual de vacinação.

Protocolo de sincronização. Os animais foram submetidos a um protocolo de sincronização da onda folicular e da ovulação, iniciado em um dia aleatório do ciclo estral denominado dia 0 (D0), por meio da inserção de um dispositivo intravaginal contendo $1,0 \mathrm{~g}$ de progesterona (Sincrogest ${ }^{\circledR}$, Ouro-Fino, São Paulo, Brasil), associado à aplicação de 2,0mg de benzoato de estradiol pela via intramuscular (im) (Sincrodiol ${ }^{\circledR}$, Ouro-Fino, São Paulo, Brasil). No D7, estes animais receberam 12,5mg de dinoprost trometamina (im) (Lutalyse ${ }^{\circledR}$, Zoetis, São Paulo, Brasil). No D9, pela manhã, foi realizada a remoção dos dispositivos intravaginais de progesterona, realizada aplicação de $0,6 \mathrm{mg}$ de cipionato de estradiol (im) (ECP ${ }^{\circledR}$,Zoetis, São Paulo, Brasil) e 300UI de gonodotrofina coriônica equina (im) (eCG, Novormon ${ }^{\circledR}$, MSD Saúde Animal, São Paulo, Brasil).

As inseminações foram realizadas no D11, 48 horas após a retirada dos implantes, empregando-se sêmen criopreservado comercial de um único touro da raça Girolando, que se enquadravam dentro dos padrões mínimos estabelecidos pelo Colégio Brasileiro de Reprodução Animal (CBRA) (Henry \& Neves 1998). No momento da IA, o sêmen foi descongelado a $37^{\circ} \mathrm{C}$ por 30 segundos. As inseminações foram efetuadas pelo mesmo técnico seguindo recomendações da Associação Brasileira de Inseminação Artificial (ASBIA 2016).

Avaliações ultrassonográficas. As avaliações ultrassonográficas foram realizados utilizando um aparelho portátil equipado com as funções modo B e doppler colorido (Mindray Z5, Shenzhen, China), para as avaliações em modo B empregou-se um transdutor transretal com frequência de 7,5Mhz. No modo doppler colorido as configurações foram ajustadas na faixa de velocidade de $6 \mathrm{~cm} / \mathrm{s}$ para detectar movimento das células sanguíneas em pequenos vasos, para este efeito utilizou-se modo power-flow, uma frequência de 4,2Mhz, com padrões de ganho de cor de $80 \%$, frequência de repetição de pulsos (PRF) de $0,7 \mathrm{KHz}$ e a profundidade de avaliação de seis centímetros. 
A avaliação do corpo lúteo foi realizada por ultrassonografia no 20 이a após inseminação. Para tanto, empregou-se a ultrassonografia em modo B para o diâmetro do CL (DCL) por meio da média aritmética entre duas mensurações na maior secção transversal do CL. As imagens luteais armazenadas foram submetidas a avaliação objetiva de acordo com Arêas (2012), onde calculou-se a área total do CL (ACL) empregando o cursor do aparelho para indicar a toda a área da circunferência do CL e para os CLs cavitários, a área da cavidade foi calculada e subtraída do área total do CL. No cálculo da área de vascularização se utilizou o cursor para verificar a VCL (Fig.1). A determinação do percentual de vascularização na área do CL foi realizada por meio da razão entre a área de vascularização e área total do CL (\%VCL). Para CLs cavitários, a área da cavidade foi calculada e subtraída da área total do CL.

Após obtenção de todas as imagens, estas foram classificadas de forma subjetiva com base na quantidade de pixels coloridos e na sua distribuição na área de secção do CL, em três grupos (Fig.1): animais com poucos pixels coloridos, baixa vascularização, restritos as áreas centrais ou periféricas do CL foram classificados como diagnóstico preditivo negativo (DPN), enquanto que animais com muitos pixels, alta vascularização, bem distribuídos na área de secção do CL e animais com vascularização intermediária foram considerados como diagnóstico preditivo positivo (DPP). Para os três grupos foram feitas as avaliações de todas as variáveis no que diz respeito a avaliação do CL e dosagem de progesteronas já citadas.

No $34^{\circ}$ dia após as inseminações, realizou-se o diagnóstico definitivo de gestação dos animais utilizando ultrassonografia em modo B, tendo como diagnóstico positivo de prenhez, a visibilização de um embrião comprovando sua viabilidade por meio da presença de batimento cardíaco.

Coleta de sangue e ensaio hormonal. Amostras de sangue foram coletadas no $20^{\circ}$ dia após inseminação para dosagem da concentração plasmática de P4, por meio da venopunção da coccígea. Todas as amostras foram coletadas em tubos de vácuo de $10 \mathrm{~mL}$ sem anticoagulante (Vacutainer ${ }^{\circledR}$, Becton, Dickinson and Company, Franklin Lakes, NJ, EUA) e imediatamente refrigeradas em caixa isotérmica contendo gelo. Posteriormente, as amostras foram centrifugadas a 3000G por 10 minutos para separação do soro, transferidas para microtubos de polietileno, devidamente identificados e armazenados a $-20^{\circ} \mathrm{C}$ até o momento da análise. As análises da concentração de P4 foram realizadas pela metodologia de quimioluminescência utilizando o Access Immunoassay Systems Progesterone (Beckman Coulter, Fullerton, CA, USA), conforme especificações do fabricante, no Laboratório de Imunologia do Instituto de Ciências da Saúde (ICS) da Universidade Federal da Bahia.

Delineamento experimental e procedimentos estatísticos. Para a análise estatística foi utilizado o programa Statistical Package for Social Science (SPSS, versão 19). Para tanto, realizou-se a seguinte sequência de análises: 1) Os dados foram avaliados segundo a normalidade utilizando o teste de Shapiro Wilk demonstrando que as variáveis apresentavam comportamento normal; 2) A média e desvio padrão das características de interesse ao estudo foram obtidas por meio da análise descritiva; 3) As diferenças entre as médias das variáveis avaliadas na ultrassonografia modo $\mathrm{B}$ (DCL e ACL), na ultrassonografia modo doppler colorido (VCL e \%VCL) e na concentração plasmática de progesterona (P4) entre fêmeas gestantes e não gestantes foram comparadas utilizando-se o teste $t$ de student. As avaliações foram realizadas considerando um nível de significância de 0,05.

O diagnóstico preditivo aos 20 dias pós IATF foi comparado ao resultado do diagnóstico definitivo aos 35 dias e os resultados foram classificados como verdadeiro positivo (VP), verdadeiro negativo (VN), falso positivo (FP) e falso negativo (FN). Foram calculadas as taxas de sensibilidade por VP/(VP + FN), especificidade por VN/(FP + VN), valor preditivo positivo por $\mathrm{VP} /(\mathrm{VP}+\mathrm{FP})$ e preditivo negativo por $\mathrm{VN} /(\mathrm{FN}+\mathrm{VN})$, a acurácia do exame foi calculada através da formula $(\mathrm{VP}+\mathrm{VN}) /(\mathrm{VP}+\mathrm{FP}+\mathrm{FN}+\mathrm{VN})$.

\section{RESULTADOS E DISCUSSÃO}

Ao serem avaliados os grupos de baixa, média e alta vascularização

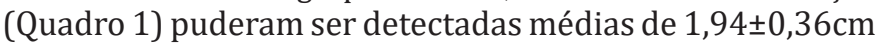
para diâmetro de CL; $3,01 \pm 0,97 \mathrm{~cm}^{2}$ para área; $0,92 \pm 0,56 \mathrm{~cm}^{2}$ para área de vascularização, 28,41\% para proporção área de

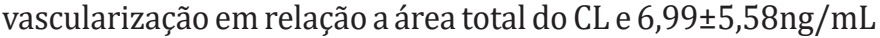
para concentração sérica de progesterona. Nas avaliações estatísticas das variáveis pode-se notar diferença significativa em todos os quesitos, entre pelo menos dois dos grupos em questão $(\mathrm{P}>0,05)$.

No que diz respeito ao diâmetro do CL, foi encontrado

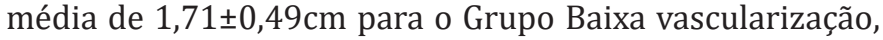
$1,96 \pm 0,30 \mathrm{~cm}$ para média e $2,05 \pm 0,21 \mathrm{~cm}$ para o Grupo de alta vascularização, denotando diferença significativa entre o primeiro e o último, assim como foi observada diferença para a Área total do CL onde os grupos com Baixa vascularização diferiu entre os grupos com Média e Alta vascularização, 2,29 $\pm 1,15$; $3,22 \pm 0,86$ e 3,33 $\pm 0,68$ respectivamente. Leal et al. (2009), realizando mensuração do diâmetro do CL no momento da transferência de embrião (D7), classificou-os em pequenos $(1,63 \pm 0,54 \mathrm{~cm})$, médios $(1,51 \pm 0,29 \mathrm{~cm})$ e grandes CLs $(2,01 \pm 0,73 \mathrm{~cm})$ e posteriormente ao exame ginecológico com auxílio de ultrassonografia em modo B, aos 45 dias, obteve-se o resultado do diagnóstico de gestação. Estes autores concluíram que o diâmetro do CL não interferiu significativamente nos índices de fertilidade, sugerindo que o diâmetro, ou mesmo a área do CL podem oferecer informações inconclusivas para
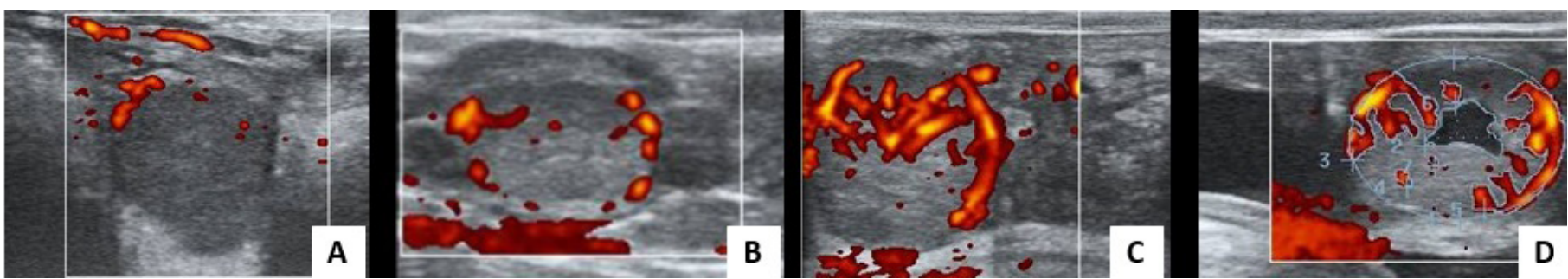

Fig.1. (A) Corpo lúteo com pouca vascularização, (B) corpo lúteo com vascularização intermediária, (C) corpo lúteo com elevada vascularização,

(D) mensuração da área total e área de vascularização do corpo lúteo, no dia 20 após a IATF. 
uma possível predição de gestação, necessitando de uma avaliação a respeito de demonstrar sua funcionalidade.

Para as variáveis de Área de Vascularização do CL e Proporção da área de vascularização, foram observadas diferenças significativas entre todos os grupos. Tal diferença demonstra uma legitimidade à classificação subjetiva nas três categorias de alta, média e baixa vascularização. Essa avaliação torna-se útil quando se realiza a ultrassonografia para diagnóstico precoce, favorecendo uma maior sensibilidade e especificidade ao teste. Comparando aos resultados de Siqueira et al. (2013), que realizaram apenas classificação dos corpos lúteos em duas categorias, baixa e alta vascularização obtiveram sensibilidade e especificidade menores, sendo observados inclusive resultados de falsos negativos.

A concentração sérica de progesterona demonstrou diferença estatística entre o grupo de baixa vascularização e os outros dois grupos, $(\mathrm{P}<0,05)$, fêmeas de Baixa vascularização

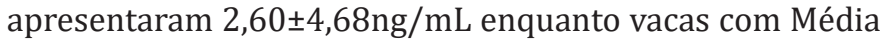
e Alta vascularização apresentaram $8,86 \pm 4,07 \mathrm{ng} / \mathrm{mL}$ e $10,52 \pm 3,74 \mathrm{ng} / \mathrm{mL}$, respectivamente. Segundo Ginther et al. (2007) e Herzog et al. (2010) a vascularização do CL avaliada por meio de ultrassonografia com Doppler colorida foi positivamente correlacionada com a secreção de P4, o que foi demonstrado no presente estudo, onde vacas que apresentaram CL maiores e mais vascularizados também apresentaram maior produção de P4.
No Quadro 2, observa-se que existiu diferença significativa $(\mathrm{P}<0,05)$ para todos os parâmetros avaliados entre os Grupos Não Gestante e Gestante.

Comparadas as áreas de vascularização do CL entre grupos não gestantes e gestantes, encontrou-se $1,78 \pm 0,46 \mathrm{~cm}^{2} \mathrm{e}$ $2,04 \pm 0,22 \mathrm{~cm}^{2}$ respectivamente. Estes resultados corroboram com Siqueira et al. (2013) quando observaram que vacas não gestantes apresentaram pouca área de vascularização quando comparadas com vacas gestantes. Assim como Guimarães et al. (2015), que constataram haver diferença significativa $(P>0,0001)$ entre a vascularização de CL de vacas gestantes ou não após 14 dias da realização de transferência de embriões realizada no D7 pós IATF. Desta forma, estes autores sugeriram que a partir da realização de imagens utilizando a ultrassonografia modo doppler colorido do CL, é possível realizar a predição da não gestação no $20^{\circ}$ dia após inseminação.

Avaliando comparativamente os resultados entre o diagnóstico preditivo aos 20 dias e o DG definitivo aos 35 pôde-se concluir que 30 dos 36 animais não gestantes foram detectados no diagnóstico preditivo resultando em uma especificidade de $83,33 \%$, assim como todos os animais gestantes foram detectados aos 20 dias, denotando uma sensibilidade de 100\% e acurácia de 91,78\%, como observado no Quadro 3. Como resultado para valores preditivos positivo e preditivo

Quadro 1. Diâmetro do corpo lúteo (DCL), área total de CL (ACL), área total de vascularização do CL (VCL), proporção da área de vascularização/área total do CL (VCL\%) e concentração de progesterona (P4), entre vacas com baixa, média e alta vascularização do CL, avaliadas por US modo doppler colorido 20 dias após a IATF

\begin{tabular}{llllllr}
\hline Grupos experimentais & $\mathrm{N}$ & DCL $(\mathrm{cm})$ & ACL $\left(\mathrm{cm}^{2}\right)$ & VCL $\left(\mathrm{cm}^{2}\right)$ & VCL\% & P4ng/mL \\
\hline Baixa vascularização & 30 & $1,71 \pm 0,49 \mathrm{~b}$ & $2,29 \pm 1,15 \mathrm{~b}$ & $0,27 \pm 0,26 \mathrm{c}$ & $10,40 \pm 6,07 \mathrm{c}$ & $2,60 \pm 4,68 \mathrm{~b}$ \\
Média vascularização & 12 & $1,96 \pm 0,30 \mathrm{ab}$ & $3,22 \pm 0,86 \mathrm{a}$ & $0,75 \pm 0,25 \mathrm{~b}$ & $23,11 \pm 2,40 \mathrm{~b}$ & $8,86 \pm 4,07 \mathrm{a}$ \\
Alta vascularização & 31 & $2,05 \pm 0,21 \mathrm{a}$ & $3,33 \pm 0,68 \mathrm{a}$ & $1,34 \pm 0,36 \mathrm{a}$ & $40,35 \pm 6,22 \mathrm{a}$ & $10,52 \pm 3,74 \mathrm{a}$ \\
Média & 73 & $1,94 \pm 0,36$ & $3,01 \pm 0,97$ & $0,92 \pm 0,56$ & $28,41 \pm 14,31$
\end{tabular}

Letras distintas divergem entre si: $\mathrm{P}=0,005 ; \mathrm{P}=0,0009 ; \mathrm{P}=0,001 ; \mathrm{P}=0,0009 ; \mathrm{P}=0,0003$.

Quadro 2. Diâmetro do corpo lúteo (DCL), área total de CL (ACL), área total de vascularização do CL (VCL), proporção da área de vascularização/área total do CL (VCL\%) e concentração de progesterona (P4) avaliados 20 dias pós-IATF, de vacas gestantes e não gestantes diagnosticadas aos 35 dias

\begin{tabular}{|c|c|c|c|c|c|c|}
\hline Grupos experimentais & $\mathrm{N}$ & DCL $(\mathrm{cm})$ & $\operatorname{ACL}\left(\mathrm{cm}^{2}\right)$ & $\operatorname{VCL}\left(\mathrm{cm}^{2}\right)$ & VCL\% & $\mathrm{P} 4(\mathrm{ng} / \mathrm{mL})$ \\
\hline Não gestantes & 36 & $1,78 \pm 0,46 b$ & $2,46 \pm 1,10 b$ & $0,43 \pm 0,41 b$ & $15,19 \pm 11,06 b$ & $3,44 \pm 4,95 b$ \\
\hline Gestantes & 37 & $2,04 \pm 0,22 \mathrm{a}$ & $3,36 \pm 0,70 \mathrm{a}$ & $1,23 \pm 0,40 \mathrm{a}$ & $36,64 \pm 8,92 \mathrm{a}$ & $10,45 \pm 3,69 a$ \\
\hline Média & 73 & $1,94 \pm 0,36$ & $3,01 \pm 0,97$ & $0,92 \pm 0,56$ & $28,41 \pm 14,31$ & $6,99 \pm 5,58$ \\
\hline
\end{tabular}

Letras distintas divergem significativamente entre si: $\mathrm{P}=0,015 ; \mathrm{P}=0,0003 ; \mathrm{P}=0,001 ; \mathrm{P}=0,0002$.

Quadro 3. Frequência de distribuição de vacas gestantes e não gestantes na predição da gestação por ultrassonografia modo doppler colorido aos 20 dias e no diagnóstico de gestação convencional aos 35 dias

\begin{tabular}{|c|c|c|c|}
\hline \multirow[t]{2}{*}{ Frequência dos resultados } & \multicolumn{2}{|c|}{$\begin{array}{c}\text { DG convencional aos } 35 \text { dias por identificação da } \\
\text { vesícula embrionária }\end{array}$} & \multirow[t]{2}{*}{ Total } \\
\hline & Gestante & Não gestante & \\
\hline \multicolumn{4}{|c|}{ DG 20 dias por avaliação de características luteais } \\
\hline Gestante & $37(\mathrm{a})$ & $6(\mathrm{~b})$ & $43(a+b)$ \\
\hline Não gestante & $0(\mathrm{c})$ & $30(d)$ & $30(c+d)$ \\
\hline TOTAL & $37(a+c)$ & $36(b+d)$ & 73 \\
\hline
\end{tabular}


negativo foram encontradas as porcentagens de $86,5 \%$ e $100 \%$ respectivamente.

Realizando avaliações seriadas do CL utilizando doppler afim de predizer a gestação nos dias 17, 19 e 21, Utt et al. (2009) demonstraram que o número de diagnósticos falso positivos diminuiu $(\mathrm{P}<0,047)$ entre os dias 17 e 19 e manteve-se inalterado entre os dias 19 e 21 . A sensibilidade não foi afetada, sendo de 82,9\%, a especificidade sofreu alterações em relação as diferentes avaliações $(\mathrm{P}<0,003)$ e aumentou $(\mathrm{P}<0,05)$ a partir do dia $17(43,2 \%)$ à 19 (54,3\%), o Valor Preditivo Negativo não foi afetado, e independentemente do momento a sensibilidade foi maior do que a especificidade e valor preditivo negativo foi maior que valor preditivo positivo $(\mathrm{P}<0,001)$. Estes autores notaram que a avaliação para predição de gestação aos dias 19 ou 21 apresentaram resultados semelhantes e satisfatórios. Em comparação aos dados do presente estudo, observou-se uma melhor confiabilidade definida pela sensibilidade de 91,3\% e especificidade de $88,8 \%$.

Contrastando com os resultados desta pesquisa, Herzog et al. (2011) caracterizaram a utilização de Doppler como não confiável para detecção precoce de gestação. 0 fator que pode explicar esta disparidade encontrada nos resultados é o momento em que foi realizada a avaliação da irrigação do CL, onde os autores supracitados realizaram a US doppler no dia 15 e 18 após a IA, enquanto no presente trabalho as imagens foram obtidas no dia 20 após IA. A diferença de tempo entre as duas avaliações pode apresentar relevância, tendo em vista que Ginther et al. (2007) demostrou existir um aumento na irrigação para o CL nos momentos próximos a luteólise o que poderia ser diagnosticado como falso positivo.

Segundo Utt et al. (2009) o método doppler colorido pode ser utilizado para predição de não gestação aos 21 dias, porém, quando usado para diagnóstico após transferências de embrião necessita cautela devido ao fato de haver perdas embrionárias tardias, ou seja, após os 21 dias. Esses animais seriam então classificados como gestantes apesar da morte embrionária, resultando em número significativo de falsos positivos. Nesse sentido, destaca-se a importância da utilização da técnica descrita como forma de incremento da produtividade quando empregada na detecção de animais não gestantes podendo realizar nova ressincronização reduzindo intervalo entre partos.

No total de 43 animais obteve-se seis animais onde o diagnóstico preditivo positivo diferiu do DG aos 35 dias. Esse fato pode ser explicado por três mecanismos: ocorrência de ciclos estrais longos, quando o CL permanece ativo por mais de 21 dias (Giordano et al. 2012), pelas perdas embrionárias que ocorrem entre os dias 21 e 35 (Ginther et al. 2007, Santos et al. 2004, Guimarães et al. 2015) e ciclos estrais curtos (9-10 dias), com a ovulação no mesmo ovário, dando origem a um CL com irrigação elevada (Taponen et al. 2003).

\section{CONCLUSÕES}

Os resultados encontrados neste trabalho caracterizam a técnica, ultrassonografia modo Doppler colorido, como uma ferramenta eficiente para realizar diagnóstico precoce de não gestação aos 20 dias após a IATF em vacas mestiças.

Pelo exposto, a técnica pode ser empregada para obter melhores resultados em relação à diminuição de intervalo entre partos e, consequentemente, influenciar no período de serviço desses animais, sendo esta uma ferramenta que possibilita ressincronização com maior brevidade, melhorando assim seu desempenho produtivo.

\section{REFERÊNCIAS}

Acosta T.J., Yoshizawa N., Ohtani M. \& Miyamoto A. 2002. Local changes in blood flow within the early and midcycle corpus luteum after prostaglandin F (2 alpha) injection in the cow. Biol. Reprod. 66(3):651-658. <http:// dx.doi.org/10.1095/biolreprod66.3.651><PMid:11870071>

Arêas V.S. 2012. Uso de Doppler colorido na avaliação funcional do corpo lúteo em bovinos. Dissertação de Mestrado, Universidade Federal do Espírito Santo, Vitória. 80p.

ASBIA 2016. Manual de Inseminação Artificial em Bovinos. ASBIA, Associação Brasileira de Inseminação Artificial.

Bollwein H., Lüttgenau J. \& Herzog K. 2012. Bovine luteal blood ow: basic mechanism and clinical relevance. Reprod. Fertil. Develop. 25(1):71-79. <http://dx.doi.org/10.1071/RD12278> <PMid:23244830>

Davis J.S., Rueda B.R. \& Spanel-Borowski K. 2003. Microvascular endothelial cells of the corpus luteum. Reprod. Biol. Endocrinol. 1(1):1-15. <http:// dx.doi.org/10.1186/1477-7827-1-1> <PMid:14613535>

Forde N., Beltman M.E., Lonergan P., Diskin M., Roche J.F. \& Crowe M.A. 2011. Oestrous cycles in Bos taurus cattle. Anim. Reprod. Sci. 124(3/4):163-169. <http://dx.doi.org/10.1016/j.anireprosci.2010.08.025><PMid:20875708>

Ginther O.J., Silva L.A., Araujo R.R. \& Beg M.A. 2007. Temporal associations among pulses of 13,14-dihydro-15-keto-PGF2alpha, luteal blood flow, and luteolysis in cattle. Biol. Reprod. 76(3):506-513. <http://dx.doi. org/10.1095/biolreprod.106.057653><PMid:17151348>

Giordano J.O., Wiltbank M.C., Guenther J.N., Pawlisch R., Bas S., Cunha A.P. \& Fricke P.M. 2012. Increased fertility in lactating dairy cows resynchronized with Double- Ovsynch compared with Ovsynch initiated $32 \mathrm{~d}$ after timed artificial insemination. J. Dairy Sci. 95(2):639-653. <http://dx.doi. org/10.3168/jds.2011-4418><PMid:22281329>

Guimarães C.R.B., Oliveira M.E., Rossi J.R., Fernandes C.A.C., Viana J.H.M. \& Palhao M.P. 2015. Corpus luteum blood flow evaluation on Day 21 to improve the management of embryo recipient herds. Theriogenology 84(2):237-241. <http://dx.doi.org/10.1016/j.theriogenology.2015.03.005> <PMid:25892341>

Henry M. \& Neves J.P. 1998. Manual para Exame Andrológico e Avaliação de Sêmen Animal. $2^{\underline{a}}$ ed. CBRA, Belo Horizonte, p.49.

Herzog K. \& Bollwein H. 2007. Application of Doppler ultrasonography in cattle reproduction. Reprod. Dom. Anim. 42(Suppl. 2):51-58. <http://dx.doi. org/10.1111/j.1439-0531.2007.00903.x><PMid:17688602>

Herzog K., Brockhan-Lüdemann M., Kaske M., Beindorff N., Paul V., Niemann H. \& Bollwein H. 2010. Luteal blood ow is a more appropriate indicator for luteal function during the bovine estrous cycle than luteal size. Theriogenology 73(5):691-697.<http://dx.doi.org/10.1016/j.theriogenology.2009.11.016> <PMid:20071016>.</jrn>

Herzog K., Voss C., Kastelic J.P., Beindorff N., Paul V., Niemann H. \& Bollwein H. 2011. Luteal blood ow increases during the first three weeks of pregnancy in lactating dairy cows. Theriogenology 75(3):549-554. <http://dx.doi. org/10.1016/j.theriogenology.2010.09.024> <PMid:20965557>

Houghton P.L., Lemenager R.P., Moss G.E. \& Hendrix K.S. 1990. Prediction of postpartum beef cow body composition using weight to height ratio and visual body condition score. J. Anim. Sci. 68:1428-1437.

Leal L.S., Oba E., Fernandes C.A.C. \& Sá Filho O.G. 2009. Evaluation of corpus luteum, uterine contractility and progesterone and estradiol plasmatic concentrations in recipients of bovine embryos. Ciênc. Anim. Bras. 10(1):174-183. <http://dx.doi.org/10.5216/cab.v10i1.448>

Mann G.E. \& Lamming G.E. 2001. Relationship between maternal endocrine environment, early embryo development and inhibition of the luteolytic 
mechanism in cows. Reproduction 121(1):175-180. <http://dx.doi. org/10.1530/rep.0.1210175><PMid:11226041>

Pinaffi F.L.V., Santos É.S., Silva M.G., Maturana Filho M., Madureira E.H. \& Silva L.A. 2015. Follicle and corpus luteum size and vascularity as predictors of fertility at the time of artificial insemination and embryo transfer in beef cattle. Pesq. Vet. Bras. 35(5):470-476. <http://dx.doi.org/10.1590/ S0100-736X2015000500015>

Santos J.E.P., Thatcher W.W., Chebel R.C., Cerri R.L.A. \& Galvão K.N. 2004. The effect of embryonic death rates in cattle on the efficacy of estrus synchronization programs. Anim. Reprod. Sci. 82-83:513-535. <http:// dx.doi.org/10.1016/j.anireprosci.2004.04.015><PMid:15271477>

Siqueira L.G.B., Areas V.S., Ghetti A.M., Fonseca J.F., Palhao M.P., Fernandes C.A.C. \& Viana J.H.M. 2013. Color Doppler flow imaging for the early detection of nonpregnant cattle at 20 days after timed artificial insemination. J. Dairy
Sci. 96(10):6461-6472. <http://dx.doi.org/10.3168/jds.2013-6814> <PMid:23958005>

Taponen J., Hjerppe P., Kopra E., Rodríguez-Martínez H., Katila T. \& Kindahl H. 2003. Premature prostaglandin F2alpha secretion causes luteal regression in GnRH-induced short estrous cycles in cyclic dairy heifers. Theriogenology 60(2):379-393. <http://dx.doi.org/10.1016/S0093-691X(03)00006-2> $<$ PMid:12749951>

Utt M.D., Johnson 3rd G.L. \& Beal W.E. 2009. The evaluation of corpus luteum blood flow using color-flow Doppler ultrasound for early pregnancy diagnosis in bovine embryo recipients. Theriogenology 71(4):707-715. <http:// dx.doi.org/10.1016/j.theriogenology.2008.09.032><PMid:18995894>

Wathes D.C., Taylor V.J., Cheng Z. \& Mann G.E. 2003. Follicle growth, corpus luteum function and their effects on embryo development in postpartum dairy cows. Reprod. Suppl. 61:219-237. PMID: 14635938. 